Mots. Les langages du politique

Le silence en politique

\title{
Les historiens face aux nouveaux usages du mot mémoire
}

Mémoire : entre « formation discursive » et " mémoire discursive »

Historians facing the new uses of the word mémoire (memory)

Los historiadores frente a los nuevos usos de la palabra mémoire (memoria)

\section{Sébastien Ledoux}

\section{CpenEdition}

Journals

Édition électronique

URL : https://journals.openedition.org/mots/21538

DOI : $10.4000 /$ mots. 21538

ISSN : 1960-6001

\section{Éditeur}

ENS Éditions

\section{Édition imprimée}

Date de publication : 16 décembre 2013

Pagination : 137-143

ISBN : 978-2-84788-527-9

ISSN : 0243-6450

Référence électronique

Sébastien Ledoux, «Les historiens face aux nouveaux usages du mot mémoire », Mots. Les langages du politique [En ligne], 103 | 2013, mis en ligne le 16 décembre 2015, consulté le 23 avril 2022. URL : http://journals.openedition.org/mots/21538; DOI : https://doi.org/10.4000/mots.21538 


\section{Les historiens face aux nouveaux usages du mot mémoire}

\section{Mémoire : entre "formation discursive" et « mémoire discursive »}

Le terme mémoire a évolué, par le biais de nouveaux usages, vers une polysémie croissante au cours des années soixante / soixante-dix. Chez les historiens, son emploi a correspondu à un objet de recherche saisi par une nouvelle école historiographique, la Nouvelle Histoire, avec notamment la notion de mémoire collective (Nora, 1978). Depuis lors et jusqu'à nos jours, ceux-ci l'utilisent pour opposer et hiérarchiser deux autorités discursives sur le passé : «Mémoire et histoire : loin d'être synonymes, nous prenons conscience que tout les oppose $»^{1}$.

En définissant de prime abord la mémoire comme un «présent du passé »², on pourrait considérer cependant que le travail de l'historien, depuis l'Antiquité, a toujours eu comme effet souhaité de rendre présent un passé pour conjurer l'oubli3. Du point de vue éthique, ce "présent du passé » est même un moteur puissant de sa pratique, si l'on suit les réflexions de Michel de Certeau, puis de Paul Ricœur à ce sujet4. La différence - essentielle - résiderait

1. Pierre Nora, «Entre mémoire et histoire », dans Nora éd., 1997, t. I, La République, p. 24.

2. Saint Augustin (354-430), Les Confessions, 1994, p. 269.

3. Hérodote (ca 484 - ca 420 av. J.-C.), considéré comme le «père de l'histoire », annonce comme suit ses Histoires, dont le titre est traduit aussi par L'Enquête : "Hérodote d'Halicarnasse présente ici les résultats de son Enquête afin que le temps n'abolisse pas le souvenir des actions des hommes et que les grands exploits accomplis soit par les Grecs, soit par les Barbares, ne tombent pas dans l'oubli. » Hérodote, Histoires, L. 1, 1985.

4. «À travers le document et au moyen de la preuve documentaire, l'historien est soumis à ce qui, un jour, fut. Il a une dette à l'égard du passé, une dette de reconnaissance à l'égard des morts, qui fait de lui un débiteur insolvable. » Ricœur, 1991, p. 253.

Université Paris 1, Centre d'histoire sociale du xxe siècle ledoux.sebastien5@gmail.com 
alors dans l'opération de recouvrement du passé, déterminée chez lui par une enquête que vient achever un récit d' «événements vrais »5.

Cependant, le terme mémoire a occupé, au cours des années quatre-vingt, une nouvelle fonction sociale qui tendrait à le situer dans un véritable « horizon d'attente " 6 , à l'échelle à la fois individuelle et collective. C'est là le paradoxe apparent de cette nouvelle forme de notre rapport au passé, qui s'est inscrite notamment dans ce mot (Hartog, 2003). Les historiens ne se trouvent pas seulement confrontés, depuis une trentaine d'années environ - soit depuis le début du «moment-mémoire »7 -, à la prééminence d'une notion mais également au pouvoir d'un mot (Foucault, 1994), l'usage qui en est fait jusqu'à aujourd'hui signalant avant tout un «usage de son pouvoir, de sa puissance d'action, de sa performativité» (Boutet, 2010, p.16). Ce mot a progressivement constitué un ensemble d'énoncés (Foucault, 1969) qui a vu la production de multiples locutions formées avec lui. Citons les plus connues : lieux de mémoire, devoir de mémoire, travail de mémoire. Mémoire est par ailleurs désormais associé à une "mémoire discursive» (Paveau, 2006) de ses locuteurs comme de ses récepteurs. Le terme est en effet «habité» (Bakhtine, 1929) par de multiples référents cognitifs renvoyant à la fois à des événements et à des catégories morales qui leur sont attachées. Les historiens ont donc été mis en concurrence dans leur autorité discursive face, à la fois, au pouvoir et à la mémoire d'un mot.

C'est au tournant des années soixante-dix/quatre-vingt que ce mot a échappé à l'autorité des historiens, non seulement comme objet scientifique, mais surtout comme notion efficiente du discours sur le passé. Le mot mémoire pris en ce sens a, de fait, largement empiété sur leur territoire de par ses usages contribuant à saisir le temps du passé pour le comprendre et en transmettre les enjeux aux contemporains. C'est aussi de cette intrusion dans leur vocation traditionnelle qu'il est question, lorsque certains d'entre eux évoquent, à partir des années quatre-vingt-dix, une « mémoire envahissante », une «vague mémorielle», une «tyrannie de la mémoire». Dans cette mise en concurrence entre deux autorités narratives du passé, le terme mémoire témoigne, depuis trente-cinq ans environ, d'une polysémie débordant largement les historiens par sa capacité à instituer du sens dans notre présent à partir de faits du passé, et à mobiliser ainsi des actions sociales, politiques, éducatives, culturelles et artistiques innombrables.

Pour sortir d'une position défensive qui peut parfois recouvrir une certaine ambivalence à l'égard de la mémoire, il apparaît nécessaire d'analyser l'his-

5. Veyne, 1996 [1971], p.16.

6. Notion développée par l'historien allemand Reinhart Koselleck pour qui «tout homme, toute communauté humaine dispose d'un espace d'expérience vécue, à partir duquel on agit, dans lequel ce qui est passé est présent ou remémoré, et des horizons d'attente, en fonction desquels on agit». Koselleck, 1987, p. 9.

7. Pierre Nora, «L'ère de la commémoration », dans Nora éd., 1997, t. III, Les France, p. 4710. 
toire de la construction socio-sémantique du désignant, plutôt que de continuer à présenter celui-ci comme référant à une entité transcendante. Il s'agit ainsi pour les historiens «d'en finir avec une certaine naïveté du momentmémoire », comme nous y invitait Marie-Claire Lavabre dès $1994^{8}$.

Autrement dit, répondre à la question «De quoi la mémoire est-elle devenue le nom ? "9 devient l'un des défis majeurs que la discipline historique se doit de relever aujourd'hui, dans un dialogue avec les autres disciplines travaillant sur un objet dont la «polysémie» (Lavabre, 2000, p.51) est l'une des caractéristiques majeures.

\section{Construction socio-sémantique du mot mémoire}

Composante essentielle du discours identitaire de communautés en pleine affirmation (régionales, culturelles, religieuses), le terme mémoire a d'abord été saisi dans les années soixante-dix, par divers acteurs sociaux appartenant à des minorités, comme un outil d'affranchissement, de libération vis-à-vis du cadre de l'État-Nation, qui faisait obstacle à ce qu'il représentait.

Dans le contexte des années quatre-vingt, qui voit émerger un nouveau statut social pour les victimes, corrélé, d'une part, à une attention au trauma (Fassin, Rechtman, 2007), et d'autre part à la vulgarisation des théories freudiennes sur le refoulement, l'appel à la mémoire a également été appréhendé comme une libération individuelle à travers la verbalisation de souvenirs enfouis dans l'inconscient.

À l'échelle collective et à partir du milieu des années quatre-vingt, le discours médiatique a présenté cette mémoire comme le moyen d'une indispensable thérapie pour une «France malade de son passé » concernant Vichy et sa complicité dans les persécutions antisémites de la seconde guerre mondiale. Ce qu'on a appelé mémoire a ainsi fait figure d'instrument politique privilégié pour rétablir la concorde nationale et pacifier le corps social en proie à l'antisémitisme et aux succès électoraux de l'extrême droite à partir du milieu des années quatre-vingt.

Les usages du terme mémoire, lors de procès particulièrement médiatisés, l'ont par ailleurs installé dans un «régime de vérité» (Foucault, 2012b, p. 158) ${ }^{10}$. La mémoire de s témoins historiques a administré la preuve de la culpabilité de criminels (Barbie, Touvier, Papon) et de la véracité de faits - la persécution

8. La sociologue considérait à cet égard que «les historiens de la mémoire ne peuvent ni échapper à la nécessité de justifier leur objet, ni éviter de s’interroger, au second degré, sur la signification même du mouvement qu'ils ont suscité, accompagné, épousé ». Lavabre, 1994, p. 481.

9. Référence au titre de l'article de M.-C. Lavabre, «De quoi la mémoire est-elle aujourd'hui le nom?», 2011, p. 35-49.

10. Le journal Le Monde (6-7 juillet 1987) titrait, au lendemain de la condamnation de Klaus Barbie pour crimes contre l’humanité : «Le procès de la mémoire». 
et l'extermination des Juifs - qualifiés de crimes contre l'humanité. À côté du travail d'historiens (Hilberg, 2006; Vidal-Naquet, 1995) réfutant les thèses négationnistes introduites dans le débat public depuis 1978 avec l'affaire Darquier de Pellepoix (Rousso, 1990; Azouvi, 2012), la mémoire des témoins s'est retrouvée au cœur des vecteurs modernes de «mémorialisation », comme la télévision (Shoah de Claude Lanzmann, émission La Marche du siècle, film du procès Barbie entre autres), les procès pour crimes contre l'humanité, la littérature de témoignage, pour énoncer le vrai (Dulong, 1998).

Ce « dispositif» (Foucault, 2012b, p. 299), dont la mémoire est l'enjeu énonciatif depuis les années quatre-vingt, renvoie à une économie, c'est-à-dire à un ensemble de pratiques, de savoirs, de mesures et d'institutions censées gouverner les comportements et les pensées des hommes.

En cela, à côté des « lois mémorielles », des pratiques commémoratives, des pratiques éducatives au sein de l'Éducation nationale, etc., le mémorial apparaît comme l'institution paradigmatique de ce dispositif. Espaces consacrés de la mémoire inscrits dans notre territoire, les mémoriaux se sont multipliés à partir de la fin des années quatre-vingt. Remplaçant les anciens mémoriaux, qui étaient davantage des lieux de recueillement en hommage aux morts, ces nouvelles institutions mémorielles concentrent différents éléments de cette économie : l'émotion du visiteur est sollicitée par une scénographie, laquelle s'appuie sur une forte présence de l'image pour lui transmettre un savoir mémorable, mais aussi lui adresser un message pour l'avenir, cela avec la participation d'historiens qui ont apporté une caution scientifique aux innombrables réalisations de mémoriaux.

La mémoire a parallèlement été habitée d'une dimension éthique, de plus en plus présente dans le contexte du déclin des théories marxistes et de la centralité nouvelle que prenait le génocide des juifs en France, mais aussi dans le monde occidental (Levy, Sznaider, 2006; Rothberg, 2009). L'injonction d'un "devoir de mémoire» concernant la Shoah s'est ainsi construite par le biais de l'adhésion à de nouvelles normes qui ont fondé un contrat social élaboré entre 1990 et 2000 par le pouvoir politique : l'État doit faire mémoire des crimes commis, au nom des valeurs des droits de l'homme. Depuis la fin de l'État-Providence dans les années soixante-dix, la puissance publique a ainsi investi la mémoire pour apporter à ses administrés une restauration de l'estime de soi, avec la mise en place de politiques de reconnaissance (Honneth, 2000 et 2004), en lieu et place d'une politique de redistribution sociale.

Enfin, la mémoire est devenue objet de créations artistiques contemporaines très variées dans les domaines de l'architecture, de la sculpture, de la peinture, de la littérature, du cinéma, de la bande dessinée, etc. Certaines œuvres ont acquis un statut de références éthico-esthétiques : Shoah de Claude Lanzmann, Si c'est un homme de Primo Levi, et plus récemment Maus d'Art Spiegelmann, par exemple. 


\section{Les historiens face à la mémoire, entre négociations et affrontements}

Face aux nouveaux emplois du mot mémoire, l'historien a été contraint de s'adapter. En venant le concurrencer sur le terrain de la production de la mémoire sociale, l'une de ses fonctions traditionnelles, ils ont mis à jour «l'ambivalence constitutive» (Revel, 2006) du métier d'historien : transmettre des connaissances et construire un rapport spécifique au présent et au passé. Derrière la posture officielle de certains, déniant à la mémoire et au «devoir de mémoire » toute capacité à construire une intelligibilité du passé, se découvrent des acteurs scientifiques qui font, dans le même temps, de la mémoire «une utilisation stratégique» (Lavabre, 2000, p.50), à la recherche de positions au sein non seulement du milieu académique, mais aussi de l'espace public ou médiatique, voire politique, le mot mémoire instituant «des effets de pouvoir propre au jeu énonciatif» (Foucault, 2012b, p.144).

D’une certaine façon, les historiens ont été conduits à entrer dans ce nouveau dispositif que représente la mémoire en mettant en avant leur rôle d' " experts du passé », à l'occasion des procès pour crimes contre l'humanité, de commissions d'enquête, de commémorations ou de créations de muséesmémoriaux (Dumoulin, 2003; Rousso, 2002). Tout en revendiquant la spécificité de leur identité à travers la formule devoir d'histoire, ils ont ainsi participé à valider scientifiquement l'«institution du sens» (Descombes, 1996) dont la mémoire était investie sur la scène sociale. Cet engagement des historiens dans des dispositifs mémoriels se négocie au cas par cas, impliquant des enjeux de pouvoir entre acteurs-historiens, ou entre eux et d'autres acteurs, selon des logiques historiographiques, de positionnement au sein du monde académique, de politiques locales, de liens avec des communautés mémorielles.

Les années deux mille ont aussi vu l'affrontement, cette fois direct, entre deux autorités narratives du passé : celle d'acteurs politiques adoptant différentes lois relatives au passé traumatique (dites lois mémorielles) au nom du «devoir de mémoire », et celle d'historiens rassemblés dans l'association Liberté pour l'histoire créée en 2005 (Rémond, 2006). La demande d'abrogation des «lois mémorielles », et d'arrêt de toute nouvelle loi qualifiant le passé, a mis en évidence une intense activité d'historiens, en particulier Pierre Nora, auprès des acteurs du pouvoir politique (parlementaires, cabinets ministériels et présidentiel).

Il existe ainsi des espaces conflictuels à différentes échelles - géographiques, institutionnelles - démontrant que le mot même de mémoire relève d'une catégorie concentrant des enjeux, des affrontements, des négociations, et cela des cabinets ministériels jusqu'au niveau le plus bas des collectivités locales. L'historien est devenu un acteur, parmi d'autres, de cette catégorisation 
du passé que l'on nomme mémoire, et qui constitue aujourd'hui une matrice puissante des relations sociales de notre présent.

\section{Références}

Azouvı François, 2012, Le mythe du grand silence. Auschwitz, les Français et la mémoire, Paris, Fayard.

BAKhtine Mikhail, 1970 [1929], La poétique de Dostoïevski, trad. du russe par I. Kolitcheff, Paris, Le Seuil.

Boutet Josiane, 2010, Le pouvoir des mots, Paris, La Dispute.

Descombes Vincent, 1996, Les institutions du sens, Paris, Minuit.

Dulong Renaud, 1998, Le témoin oculaire. Les conditions sociales de l'attestation personnelle, Paris, EHESS.

Dumoulin Olivier, 2003, Le rôle social de l'historien. De la chaire au prétoire, Paris, Albin Michel.

FASSIN Didier, RECHTMAN Richard, 2007, L'empire du traumatisme. Enquête sur la condition de victime, Paris, Flammarion.

Foucault Michel, 2012a [1969], L'archéologie du savoir, Paris, Gallimard (Tel).

— 2012b [1994], Dits et écrits, vol. Il, Paris, Gallimard (Quarto).

HARTOG François, 2002, Des régimes d'historicité. Présentisme et expériences du temps, Paris, Seuil.

HÉROdOTE, 1985, Histoires, 1. I, trad. A. Barguet, Paris, Gallimard.

HILBERg Raul, 2006 [1988], La destruction des Juifs d'Europe, Paris, Gallimard (Folio histoire).

HONNETH Axel, 2000, La lutte pour la reconnaissance, trad. de l'allemand par P. Rusch, Paris, Cerf.

- 2004, "La théorie de la reconnaissance : une esquisse», Revue du MAUSS, n²3, p. $133-136$

KOSELleck Reinhart, 1987, «Temps et histoire», Romantisme, n56.

LAVABRe Marie-Claire, 1994, "Usages du passé, usages de la mémoire», Revue française de science politique, nº3, p.480-493.

- 2000, «Usages et mésusages de la notion de mémoire», Critique internationale $\mathrm{n}^{0} 7, \mathrm{p} .48-57$.

- 2011, «De quoi la mémoire est-elle aujourd'hui le nom?», L'enigma della memoria collettiva. Politica, istituzioni, conflitti, L. Migliorati, L. Mori éd., Vérone, QuiEdit, p. 35-49.

LEDoux Sébastien, 2012, «Écrire une histoire du “devoir de mémoire”, Le Débat, nº170, p. $175-185$.

- à paraître (2013), "Les lieux d'origine du devoir de mémoire», Conserveries mémorielles, revue numérique, Université de Laval (Canada) / IHTP.

LE Goff Jacques, NoRA Pierre éd., 1986 [1974], Faire de l'histoire, Paris, Gallimard (Folio Histoire). 
Levy Daniel, Sznaider Natan, 2006, The Holocaust and Memory in a Global Age, Philadelphia Temple University Press.

Nora Pierre, 1978, « Mémoire collective », La Nouvelle Histoire, J. Le Goff éd., Paris, Retz, p. 398-401.

— éd., 1997 [1984-1993], Les lieux de mémoire, Paris, Gallimard (Quarto).

Paveau Marie-Anne, 2006, Les prédiscours. Sens, mémoire, cognition, Paris, Presses de la Sorbonne nouvelle.

RÉmond René, 2006, «L'Histoire et la Loi », Études, n²404, p. 763-773.

Ricceur Paul, 1991 [1983], Temps et récit, t. I, L'intrigue et le récit historique, t. III, Le temps raconté, Paris, Le Seuil (Points Essais).

- 2003 [2000], La mémoire, l'histoire, l'oubli, Paris, Le Seuil (Points Essais).

ROTHBERg Michael, 2009, "Multidirectional memory and the universalization of the Holocaust», Remembering the Holocaust. A Debate, J. Alexander et al. éd., NewYork, Oxford University Press.

ReVEL Jacques, 2006, Un parcours critique. Douze exercices d'histoire sociale, Paris, Gaalade.

Rousso Henry, 1990 (rééd.), Le syndrome de Vichy de 1944 à nos jours, Paris, Le Seuil (Points Histoire).

- 2002, "L'expertise des historiens dans les procès pour crimes contre l'humanité », Barbie, Touvier, Papon. Des procès pour la mémoire, J.-P. Jean, D. Salas éd., Paris, Autrement, p. 58-70.

Saint Augustin, 1994, Les Confessions, trad. J. Trabucco, Paris, Garnier Flammarion.

VeYne Paul, 1996 [1971], Comment on écrit l'histoire, Paris, Le Seuil (Points Histoire).

VIDAL-NAQUet Pierre, 1987, Les assassins de la mémoire, Paris, Le Seuil (Points Essais), rééd. 1995. 